\title{
Effect of Accelerated Thermal Ageing on the Selective Solar Thermal Harvesting Properties of Multiwall Carbon Nanotube/Nickel Oxide Nanocomposite Coatings
}

\author{
Kittessa T. Roro, ${ }^{1}$ Bonex Mwakikunga, ${ }^{2}$ Ngcali Tile, ${ }^{1,3}$ Brian Yalisi, ${ }^{1,3}$ and Andrew Forbes ${ }^{1,3}$ \\ ${ }^{1}$ CSIR National Laser Centre, P.O. Box 395, Pretoria 0001, South Africa \\ ${ }^{2}$ DST/CSIR National Centre for Nano-Structured Materials, P.O. Box 395, Pretoria 0001, South Africa \\ ${ }^{3}$ School of Physics, University of KwaZulu Natal, Private Bag X54001, Durban 5000, South Africa
}

Correspondence should be addressed to Kittessa T. Roro, kroro@csir.co.za and Bonex Mwakikunga, bmwakikunga@csir.co.za

Received 6 February 2012; Accepted 27 March 2012

Academic Editor: Mohamed Sabry Abdel-Mottaleb

Copyright ( $) 2012$ Kittessa T. Roro et al. This is an open access article distributed under the Creative Commons Attribution License, which permits unrestricted use, distribution, and reproduction in any medium, provided the original work is properly cited.

\begin{abstract}
Varying amounts of dispersed multiwalled carbon nanotubes in $\mathrm{NiO}$ have been used to develop composites that absorb the solar energy very well but lose very little through emission. Determination of absorptance, $\alpha_{\text {sol }}$, and emissivity, $\varepsilon_{\text {ther }}$, from such selective solar absorbers shows that the optimum efficiency of $71 \%$ can be attained when about $10 \mathrm{mg}$ of MWCNTs are composited with $\mathrm{NiO}$. One such absorber was subjected to thermal ageing tests. The performance criterion (PC) limit for passing the test when simulated for 25 years is $(-\Delta \alpha+0.25 \Delta \varepsilon) \leq 0.05$. It was found that the typical absorber had a PC value of -0.01 . This value is much better than the passing limit. Raman spectra of the typical absorber before and after the thermal ageing test showed a reduced intensity in the $\mathrm{D}$ and $\mathrm{G}$ bands of disordered and graphitic carbon, respectively but an enhancement of the NiO bands indicating loss of carbon atoms due to thermal ageing tests. Simple equations are derived determining the proportion of carbon atoms that are lost and the proportion of carbon atoms that remains in the absorber; both of these are in agreement with the original carbon composition before the thermal ageing test. It is reported that the typical absorber will retain $63 \%$ of the carbon after 25 years.
\end{abstract}

\section{Introduction}

Due to increase in energy cost, environmental concern, and a need for offgrid power systems, scientists and engineers have been working on ways to harness the energy from the sun. Solar energy is cheap, clean and widely available on the earth. The solar power reaching the earth is nearly four orders of magnitude greater than the power consumption globally. Out of the power of the sunlight reaching the surface of the earth, of $1.2 \times 10^{5} \mathrm{TW}$, man only uses about 13 TW [1]. If all the energy of the sun striking the earth is converted to a useful form of energy with maximum efficiency, the annual energy consumption by humans $(4.6 \times$ $10^{20}$ Joule/year) could be satisfied within one hour [1]. This clearly suggests a greater need in harvesting solar energy.

The two major technologies widely used for converting solar energy are solar thermal conversion and solar cell electricity generation. Solar thermal energy is a technology for harnessing solar energy for thermal energy (heat). Solar thermal collectors for water heating use a surface that absorb sunlight and convert it to heat. Solar absorber surfaces are usually black, since dark surfaces demonstrate a particularly high degree of light absorption. The level of absorption indicates the amount of short-wave solar radiation being absorbed, that is, not being reflected. As the absorber warms up to a temperature higher than the ambient temperature, it gives off a great part of the received solar energy in the form of long-wave heat rays. The ratio of absorbed energy to emitted heat is an indication of emission.

In order to reduce energy loss through heat emission, a spectrally selective surface is used. The surface allows the conversion of a high proportion of the solar radiation into heat, while reducing the emission of heat. Thus, the requirements for the optical properties of a selective solar 
absorber coating for a high efficient solar-thermal energy conversion are a high spectral absorption (that means a low spectral reflectance) in the wavelength range of the solar radiation combined with low spectral absorption (that means a high spectral reflectance) in the wavelength range of the thermal radiation to reduce radiative heat losses [2]. To achieve this, the solar absorptance, $\alpha_{\text {sol }}$, and thermal emittance $\varepsilon_{\text {ther }}$, of the absorbing surface must be optimized.

Carbon-based nanostructured composite films consisting of nanosize particles embedded in metal oxide host matrix have attractive properties for selective solar absorber coatings to be used for solar water heating and cooking applications [3-6]. Recently, Katumba et al. [4, 5] have compared carbon embedded in three different metal oxides on Al substrates $\left(\mathrm{SiO}_{2}, \mathrm{ZnO}\right.$, and $\left.\mathrm{NiO}\right)$. Among the three, carbon in $\mathrm{NiO}$ matrix has shown superior optical properties $[4,5]$.

Much as the composite material (metal oxides and carbon) may show good selectivity, the usual question to ask is "how long does the material sustain these good properties of high absorption $\alpha_{\text {sol }}$ and low emittance $\varepsilon_{\text {ther? }}$ ?" For this reason, it is important to determine the values of $\alpha_{\text {sol }}$ and $\varepsilon_{\text {ther }}$ versus time in years. To quickly get this information in a shorter time than years, the so-called accelerated ageing tests simulated to cover a period of 25 years are employed [7].

In this work, we present accelerated thermal ageing test results on $\mathrm{NiO}$-carbon nanotube selective solar absorbing composite coated on $\mathrm{Al}$ substrate. In the experimental section, we present the conditions for the accelerated thermal ageing test, and we also present the conventional minimum requirements for whether the material passes the test or not.

\section{Experimental}

2.1. Materials. MWCNTs (synthesized by the CVD method, purity $>90 \%, 110-170 \mathrm{~nm}$ in diameter) and all other reagents were purchased from Sigma-Aldrich and used as received without further purification.

2.2. Functionalization of MWCNTs. In order to minimize tube damage, a relatively low acid concentration and exposure time, respectively, were used. The CNTs in the form of powder were added to a mixture of concentrated $\mathrm{HNO}_{3}$ and $\mathrm{H}_{2} \mathrm{SO}_{4}$ solution (0-50\%) and refluxed at $120^{\circ} \mathrm{C}$ for $2 \mathrm{hrs}$ to treat the CNTs with the acids. Subsequently, the acidic solution was passed through a filter to collect the acid-treated CNTs. The acid-treated CNTs were washed several times with distilled water and dried at $110^{\circ} \mathrm{C}$ overnight.

2.3. Substrate Preparation. The absorbing films were coated on rough highly reflecting aluminium substrates. The substrates were cut into a $55 \times 55 \mathrm{~mm}^{2}$ size and cleaned before deposition. The precleaning process involved cleaning the substrates with aqueous detergent and distilled water in order to remove the grease. Due to poor adhesion to the aluminium surfaces (uncleaned or cleaned with soap and water), the substrates were etched. The precleaned substrates were thoroughly rinsed and dipped in a phosphoric acid bath at $60^{\circ} \mathrm{C}$ for about 30 minutes to remove the protective oxide layer. They were then thoroughly rinsed using distilled water to remove the acid. Finally, they were blown dry with a $\mathrm{N}_{2}$ and coated immediately.

2.4. Nanocomposite Preparation. The preparation of the solution was adopted from a previous experiment [8]. The $\mathrm{NiO}$ precursor solution was prepared by dissolving $7.4 \mathrm{~g}$ of nickel acetate $\left[\left(\mathrm{NiAc}_{2}\right), \mathrm{Ni}\left(\mathrm{CH}_{3} \mathrm{COO}\right)_{2} \cdot 4 \mathrm{H}_{2} \mathrm{O}\right]$ in $50 \mathrm{~mL}$ of absolute ethanol [(EtOH), $\left.\mathrm{CH}_{3} \mathrm{CH}_{2} \mathrm{OH}\right]$ followed by magnetic stirring at room temperature for $2 \mathrm{hrs}$. The solvent, ethanol, is evaporated from the mixture by heating at $50^{\circ} \mathrm{C}$ for about $20 \mathrm{~min}$ until the final solution is reduced to half to make the solution more viscous. Thereafter, $6.3 \mathrm{~g}$ of diethanolamine [(DEA), $\left.\mathrm{NH}\left(\mathrm{CH}_{2} \mathrm{CH}_{2} \mathrm{OH}\right)_{2}\right]$ was added as a chelating agent and then stirred further for $5 \mathrm{~min}$. Following this, $2 \mathrm{~g}$ of polyethylene glycol [(PEG), $\mathrm{HO}\left(\mathrm{CH}_{2} \mathrm{CH}_{2} \mathrm{O}\right) \mathrm{nH}$ ], a structure directing template, was added to the $\mathrm{NiO}$ matrix precursor sol and stirred for $2 \mathrm{~min}$. For the multiwall carbon nanotube precursor solution, different concentrations (10, $50,100,150$, and $200 \mathrm{mg}$ ) of functionalized MWCNTs (fMWCNTs) were added into $6 \mathrm{~mL}$ deionized water and stirred at room temperature prior to mixing with the $\mathrm{NiO}$ matrix precursor solutions. The oxide and multiwall carbon nanotube precursor solutions were mixed, and the resultant solution was then further stirred for $24 \mathrm{hrs}$ until the formation of a sol. The final solution was spin-coated at 5000 RPM on top of the precleaned aluminium substrates. Thereafter, the samples were heat-treated at $450^{\circ} \mathrm{C}$ in nitrogen ambient for one hour.

2.5. Nanocomposite Analysis. The near-normal spectral reflectance of the samples was measured in the $0.3-2.5 \mu \mathrm{m}$ wavelength range with a Perkin Elmer Lambda 900 spectrophotometer. A spectralon sample was used for reference spectrum measurements (a spectralon sample is a popular white reference standard material used during reflectance measurements to maximize data quality). The reflectance measurements were used to calculate solar absorptance, $\alpha_{\text {sol }}$, of the samples using (1) [2]:

$$
\alpha_{\text {sol }}=\frac{\int_{0.3}^{2.5} I_{\text {sol }}(\lambda)(1-R(\lambda)) d \lambda}{\int_{0.3}^{2.5} I_{\text {sol }}(\lambda) d \lambda},
$$

where $I_{\text {sol }}(\lambda)$ is the incident solar spectrum and $R(\lambda)$ is the reflectance of the material. The thermal emittance, $\varepsilon_{\text {ther }}$, of each coating was measured using an emissometer model AE1 from Device and Service (USA) company, with an accuracy of \pm 0.01 emittance units. The device has a thermopile radiation detector, which is heated at $82^{\circ} \mathrm{C}$. Before taking the measurement, the emissometer is calibrated using two standards, one having an emittance of 0.88 , and the other having an emittance of 0.05 .

The thermogravimetric analysis (TGA) was done on Perkin-Elmer TGA 4000 thermogravimetric analyzer. Approximately $100 \mathrm{mg}$ final mixture of $\mathrm{MWCNT} / \mathrm{NiO}$ precursor solution was placed in open $190 \mu \mathrm{L}$ alumina pan and heated from $25^{\circ} \mathrm{C}$ to $980^{\circ} \mathrm{C}$ at a heating rate of $5^{\circ} \mathrm{Cmin}^{-1}$ in $\mathrm{N}_{2}$ atmosphere (flow rate $50 \mathrm{~mL} \mathrm{~min}^{-1}$ ). 


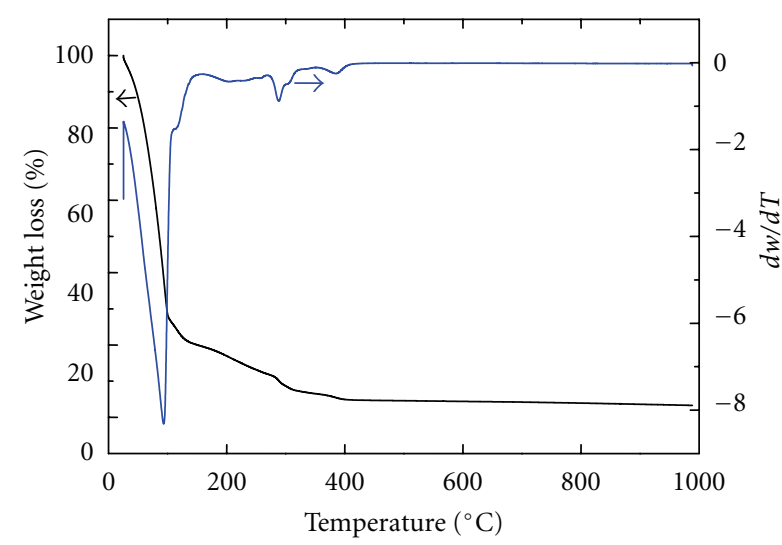

FIGURE 1: Thermogravimetric analysis (TGA) and differential TGA (DTGA) of the typical MWCNT-NiO precursor sol showing that a temperature of more than $400^{\circ} \mathrm{C}$ is necessary for decomposition of the precursor to the final composite.

The surface morphology of the coatings was investigated using a ZEISS ULTRA plus FEG-SEM scanning electron microscopy. Raman spectroscopy was conducted using a Jobin-Yvon T64000 Raman spectrograph with a $514.5 \mathrm{~nm}$ line from an argon ion laser. The T64000 was operated in single-spectrograph mode, with the 1800 lines $/ \mathrm{mm}$ grating and a 100x objective on the microscope.

2.6. Accelerated Thermal Ageing Test. In order to simulate the expected service life time of the coating of 25 years, the IEA SHC task X [7] has defined a performance criterion (PC):

$$
\mathrm{PC}=-\Delta \alpha+0.25 \Delta \varepsilon, \quad \mathrm{PC}= \begin{cases}\leq 0.05 & \text { PASS } \\ >0.05 & \text { FAIL }\end{cases}
$$

where $\Delta \alpha$ is the change in normal absorptance, and $\Delta \varepsilon$ is the change in normal emittance after and before the test calculated, thus:

$$
\begin{aligned}
\Delta \alpha & =\left(\alpha_{\text {sol }}\right)_{\text {after test }}-\left(\alpha_{\text {sol }}\right)_{\text {before test }}, \\
\Delta \varepsilon & =\left(\varepsilon_{\text {thermal }}\right)_{\text {after test }}-\left(\varepsilon_{\text {thermal }}\right)_{\text {before test }} .
\end{aligned}
$$

If PC is $\leq 0.05$, the sample passes the test. For accelerated thermal ageing test, the sample was exposed to a temperature of $250^{\circ} \mathrm{C}$ for 200 hours in air.

\section{Results and Discussion}

3.1. Thermal Characterization. A typical TGA and differential TGA (DTGA) spectra showing the different decomposition pattern of the final $\mathrm{f}-\mathrm{MWCNT} / \mathrm{NiO}$ precursor solution as a function of temperature is depicted in Figure 1. The observed weight loss corresponds to different regions indicating the intervals of the thermal decomposition of the final precursor solution. An initial mass loss of $~ 70 \%$ may be attributed to loss of ethanol and water. The second mass loss appears as a shoulder on the DTGA spectrum and represents a mass loss of $\sim 10 \%$ at around $128^{\circ} \mathrm{C}$ could be due to decomposition of undecomposed nickel acetate or nickel

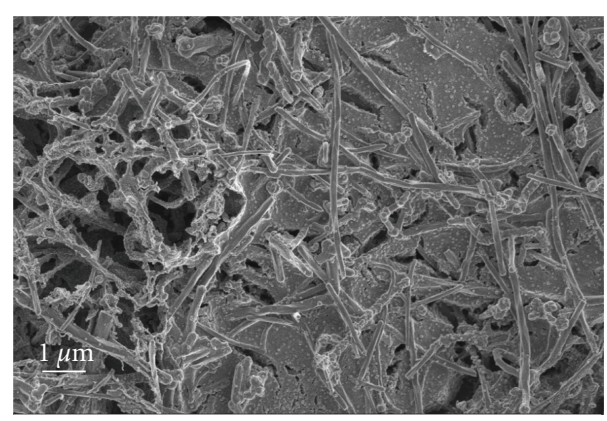

FIGURE 2: Scanning electron microscope image of a typical sample of MWCNT dispersed in NiO.

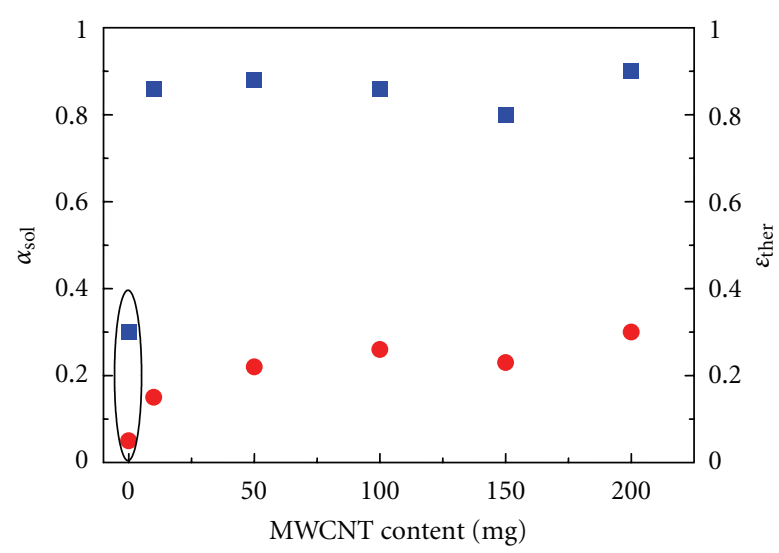

FIGURE 3: Absorptance (square) and emissivity (circle) of several MWCNT/NiO films of varying concentration of MWCNTs.

acetate/diethanolamine complexes. The third, fourth, and fifth mass losses of $\sim 10 \%, 5 \%$, and $3 \%$ at $125^{\circ} \mathrm{C}, 288^{\circ} \mathrm{C}$, and $385^{\circ} \mathrm{C}$ can be attributed to the decomposition of the organic molecules. Taking into consideration the above observation, it has been chosen to heat treat the samples at $450-550^{\circ} \mathrm{C}$.

3.2. Surface Morphology. Successful dispersion of the MWCNT in the NiO matrix was achieved. This is shown by a scanning electron microscopy image of the surface morphology of a typical sample of the MWCNT/NiO composite film on Al substrate given in Figure 2.

3.3. Optical Characterization. The solar absorptance $\alpha_{\text {sol }}$ calculated using (1) from reflectance spectra and thermal emittance $\varepsilon_{\text {ther }}$ at $82^{\circ} \mathrm{C}$ measured using the emissometer as a function of f-MWCNT concentrations is shown in Figure 3. It can be seen from the figure that for the sample with no CNTs (quasipure $\mathrm{NiO}$ obtained by heat treating in air) the solar absorptance value is 0.3 . Addition of small mass of CNTs followed by heat treatment in ambient nitrogen drastically increases the solar absorptance of the composite. Further increase in the concentration of CNTs did not change the solar absorptance value. However, the thermal emittance value increases with the increase in concentration of CNTs. 


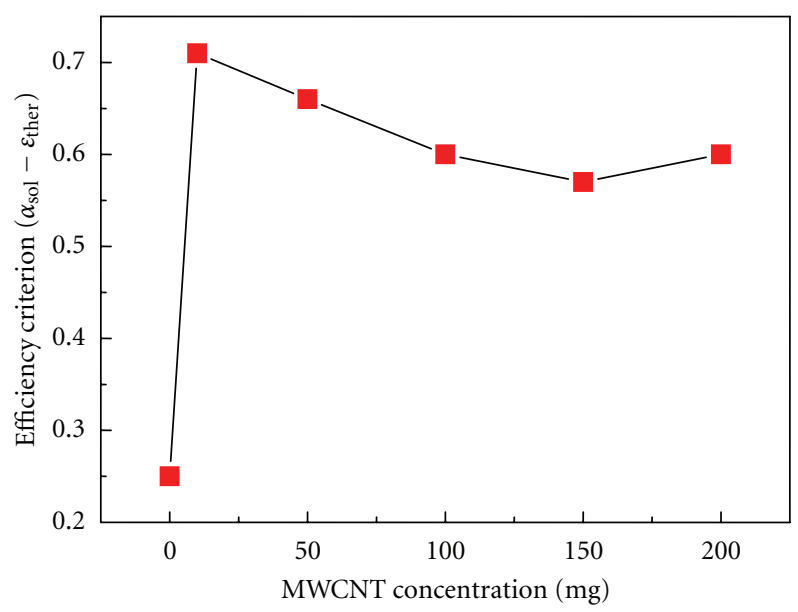

Figure 4: A plot of the photothermal conversion efficiency against the MWCNT concentration. The efficiency of light-to-heat conversion is small when the concentration CNT is near zero but increases rapidly when just less than $10 \mathrm{mg}$ of CNTs are added to same mass of $\mathrm{NiO}$. Addition of CNT beyond $10 \mathrm{mg}$ decreases the conversion efficiency.

The photothermal conversion efficiency of the absorber material can be calculated by [6]:

$$
\eta=\alpha_{\text {sol }}-\varepsilon_{\text {ther }}
$$

The photothermal conversion efficiency of the $\mathrm{f}$ MWCNT/NiO composites as a function of MWCNT concentration is shown in Figure 4. The efficiency is small when the concentration CNT is quasi zero but increases rapidly when just less than $10 \mathrm{mg}$ of CNTs are added to same mass of NiO. Addition of CNT beyond $10 \mathrm{mg}$ decreases the conversion efficiency. This indicates that only $10 \mathrm{mg}$ MWCNTs in $\mathrm{NiO}$ is important in optimising the photothermal conversion efficiency. The optimum efficiency is found to be $71 \%$. The efficiency is not improved by continually increasing the dose of MWCNT to the NiO. In fact, above this critical dose, one can refer to the situation as "poisoning" of the composite.

3.4. Accelerated Thermal Ageing Study. Since it is impractical to obtain the real thermal stability of a solar absorber for its service life time, indoor-accelerated thermal ageing test which simulate the actual scenario has to be conducted to determine whether the coating lasts or not for the duration of the anticipated service time. The reflectance spectra of the samples as deposited and after subjection to the heat treatment are shown in Figure 5. A spectrum of the solar radiation at atmospheric mass AM 1.5 is added to the graph. The red line represents the total reflectance of the absorber coating of the as deposited sample. This data gave a solar absorptance $\alpha_{\text {sol }}$ of 0.9 and the thermal emittance $\varepsilon_{\text {ther }}$ (at $82^{\circ} \mathrm{C}$ ) of 0.29 .

For the accelerated thermal ageing test, the same sample has been heated in air at $250^{\circ} \mathrm{C}$ for $200 \mathrm{hrs}$. The reflectance spectrum is added to Figure 5. From the solar spectrum, absorptance of the sample is calculated. The emittance values

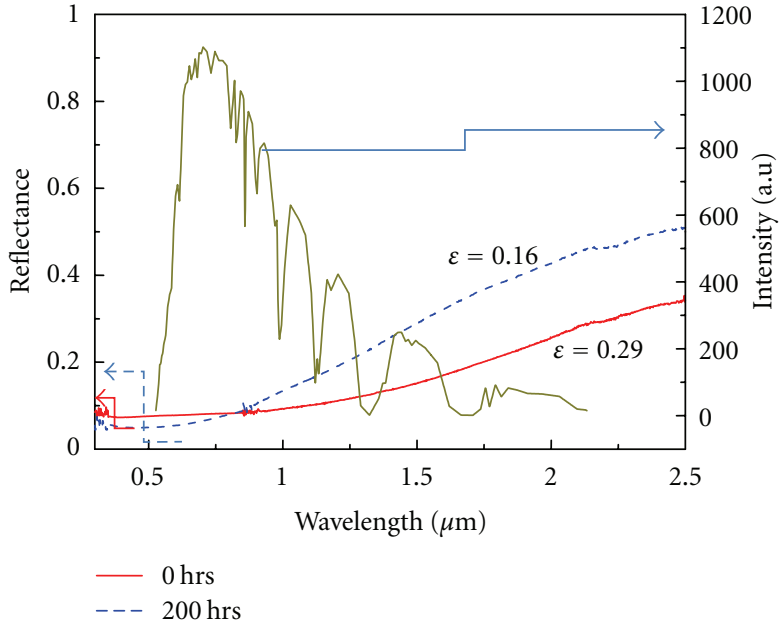

Figure 5: Reflectance of the typical selective solar absorber sample before and after thermal ageing test compared to the solar radiation spectrum. From the solar spectrum, absorptance of the sample is calculated. The emittance values as determined from the commercial emissometer are indicated on the reflectance spectra before and after thermal ageing tests.

as determined from the commercial emissometer are indicated on the reflectance spectra before $\left(\varepsilon_{\text {ther }}=0.29\right)$ and after $\left(\varepsilon_{\text {ther }}=0.16\right)$ thermal ageing tests. It can be seen from the spectrum that the reflectance decreased slightly in the solar range $(\lambda<1 \mu \mathrm{m})$ after heat treatment, whereas it increases in the NIR region. The solar absorptance for this sample is 0.88 . The change in solar absorptance $\Delta \alpha=-0.02$. Thus, the performance criterion (PC) calculated using equation 2 is -0.012 . This implies that the sample passes the test.

\subsection{Raman Spectroscopy Before and After Thermal Ageing} Tests. The structural stability of $\mathrm{f}-\mathrm{MWCNT/NiO}$ composite coatings was studied using micro-Raman spectroscopy. The Raman spectroscopy of the as-deposited sample and sample heat-treated at $250^{\circ} \mathrm{C}$ in air for $200 \mathrm{hrs}$ are shown in Figure 6. It can be observed from the Raman spectrum of the as deposited sample that the sample shows four distinct peaks at $521.5,1110.8,1358.0$, and $1583.2 \mathrm{~cm}^{-1}$. The peaks at 521.5 and $1110.8 \mathrm{~cm}^{-1}$ are ascribed to the $\mathrm{NiO}$ phonons. The other two peaks are due to carbon: a $G$ peak at around $1583.2 \mathrm{~cm}^{-1}$ due to optical zone centre vibrations ( $\mathrm{E}_{2 \mathrm{~g}}$ mode) in graphite of aromatic rings, and a D peak at around $1358 \mathrm{~cm}^{-1}$, due to the disorder activated optical zone edge modes of microcrystalline graphite sheets $[8,9]$. After the heat treatment, the $\mathrm{NiO}$ phonon enhanced, whereas the $\mathrm{D}$ and $G$ peaks were suppressed. This can be explained by slight oxidation of carbon. This minimal change in structure is expected after exposing the sample to this extreme condition. It is interesting to mention that the shape of the Raman spectrum did not change significantly.

As can be seen in Figure 6, Raman spectroscopy of the MWCNT/NiO sample, before and after the accelerated thermal ageing test, shows an enhancement in the $\mathrm{NiO}$ phonon at the expense of the intensity of the D and G bands of the carbon. 
TABLE 1: Tabulation of phonon peak parameters for a typical sample before and after the ageing test.

\begin{tabular}{|c|c|c|c|c|c|c|c|c|}
\hline \multirow{2}{*}{ Peak assignment } & \multicolumn{3}{|c|}{ Before thermal ageing test } & \multicolumn{5}{|c|}{ After thermal ageing test } \\
\hline & $\begin{array}{c}\text { Area, } \int I(\omega) d \omega \\
\left(\mathrm{cm}^{-1}\right)\end{array}$ & $\begin{array}{c}\text { Centre, } \omega_{0} \\
\left(\mathrm{~cm}^{-1}\right)\end{array}$ & $\begin{array}{l}\text { Width, } \Gamma \\
\left(\mathrm{cm}^{-1}\right)\end{array}$ & $\begin{array}{l}\text { Intensity, } I \\
\text { (arb. units) }\end{array}$ & $\begin{array}{c}\text { Area, } \int I(\omega) d \omega \\
\left(\mathrm{cm}^{-1}\right)\end{array}$ & $\begin{array}{c}\text { Centre, } \omega_{0} \\
\left(\mathrm{~cm}^{-1}\right)\end{array}$ & $\begin{array}{l}\text { Width, } \Gamma \\
\left(\mathrm{cm}^{-1}\right)\end{array}$ & $\begin{array}{l}\text { Intensity, } I \\
\text { (arb. units) }\end{array}$ \\
\hline $\mathrm{NiO}$ & 18051 & 524.3 & 84.2 & 171.1 & 61669 & 502 & 122.9 & 400.4 \\
\hline $\mathrm{NiO}$ & 73115 & 1121.2 & 170.6 & 341.9 & 47305 & 1109.8 & 143.8 & 262.5 \\
\hline $\mathrm{D}$ & $3.4 \times 10^{5}$ & 1374.9 & 182.2 & 1475.9 & $2.00 \times 10^{5}$ & 1361.9 & 143.3 & 1115.4 \\
\hline G & $2.2 \times 10^{5}$ & 1580.8 & 101.9 & 1693.4 & $1.50 \times 10^{5}$ & 1579.6 & 107 & 1118.5 \\
\hline
\end{tabular}

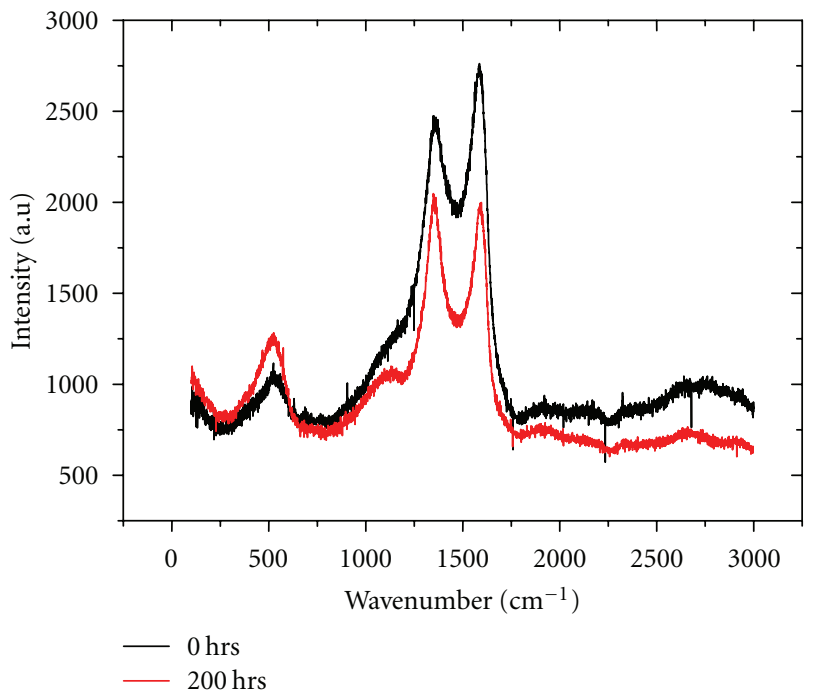

Figure 6: Fingerprint Raman spectra for a typical $(\mathrm{MWCNT})_{x}(\mathrm{NiO})_{1-x}$ before and after thermal ageing test. The main phonon peaks due to $\mathrm{NiO}\left(\sim 524\right.$ and $\left.\sim 1100 \mathrm{~cm}^{-1}\right)$ and carbon $\left(\omega_{\mathrm{D}} \sim 1370 \mathrm{~cm}^{-1}\right.$ and $\left.\omega_{\mathrm{G}} \sim 1580 \mathrm{~cm}^{-1}\right)$ clearly show a loss of carbon after ageing test.

The main phonon peaks due to $\mathrm{NiO}(\sim 524$ and $\sim$ $\left.1100 \mathrm{~cm}^{-1}\right)$ and carbon $\left(\omega_{\mathrm{D}} \sim 1370 \mathrm{~cm}^{-1}\right.$ and $\omega_{\mathrm{G}} \sim$ $1580 \mathrm{~cm}^{-1}$ ) clearly show a loss of carbon after ageing test. This is a qualitative evidence that the film loses carbon after the ageing tests, thereby rendering the film composition more $\mathrm{NiO}$-rich than before. In other words, in the initial composition, for instance, of $\operatorname{MWCNT}_{x}(\mathrm{NiO})_{1-x}$, thermal ageing tests make $x$ tend to zero. In order to quantify the proportion of carbon lost, $\beta$, and the proportion of carbon remaining in the sample, $1-\beta$, after the ageing test, one can proceed as follows: if one can represent carbon loss due to thermal ageing test as the difference between the area under the $D$ and $G$ band of graphitic carbon, thus:

$$
\Delta(\mathrm{MWCNT})=\int_{\mathrm{CNT}}^{\text {before }} I(\omega) d \omega-\int_{\mathrm{CNT}}^{\text {after }} I(\omega) d \omega
$$

and the relative "increase" in the proportion accorded to $\mathrm{NiO}$ due to the lost carbon atoms as follows:

$$
\Delta(\mathrm{NiO})=\int_{\mathrm{NiO}}^{\text {after }} I(\omega) d \omega-\int_{\mathrm{NiO}}^{\text {before }} I(\omega) d \omega
$$

One can represent the proportion of the carbon lost to the original total carbon composition [10], thus:

$$
\begin{aligned}
\beta & =\frac{\Delta(\text { MWCNT })}{\left(\int_{\mathrm{CNT}} I(\omega) d \omega\right)_{\text {before }}}=1-\frac{\left(\int_{\mathrm{CNT}} I(\omega) d \omega\right)_{\mathrm{after}}}{\left(\int_{\mathrm{CNT}} I(\omega) d \omega\right)_{\mathrm{before}}} \\
& =1-\frac{\left(\int_{\mathrm{D}} I(\omega) d \omega+\int_{\mathrm{G}} I(\omega) d \omega\right)_{\mathrm{after}}}{\left(\int_{\mathrm{D}} I(\omega) d \omega+\int_{\mathrm{G}} I(\omega) d \omega\right)_{\text {before }}} .
\end{aligned}
$$

The proportion of carbon remaining in the film after the thermal ageing test, $1-\beta$, should be proportional to the photo-thermal conversion efficiency of the aged sample. Thus, assuming a linear correlation between them, one can write:

$$
1-\beta=\frac{\left(\int_{D} I(\omega) d \omega+\int_{G} I(\omega) d \omega\right)_{\text {after }}}{\left(\int_{D} I(\omega) d \omega+\int_{G} I(\omega) d \omega\right)_{\text {before }}}=K\left(\alpha_{\text {sol }}-\varepsilon_{\text {ther }}\right),
$$

where $K$ is proportionality constant.

The areas under the peaks in the Raman spectra were determined by peak fitting. Peak fitting was performed in Origin software. A multipeak fit subroutine was employed to fit the Gaussians to all the phonon peaks present in the whole-sample spectrum. The fitting extracted several parameters for each phonon peak including the area under the peak, the zone centre of the phonon, $\omega_{0}$, the full width at half maximum of the peak, $\Gamma$, and the maximum intensity of such a phonon peak, $I_{\max }$. These data are tabulated in Table 1.

From the Tuinstra and Koenig work [11], the ratio of the intensities of the D and $G$ bands in carbon allotropes can be used to estimate the grain sizes or intergrain boundary distances, $L_{a}$, thus:

$$
\frac{I_{\mathrm{D}}}{I_{\mathrm{G}}}=\frac{44 \mathrm{~nm}}{L_{a}}
$$

$I_{\mathrm{D}} / I_{\mathrm{G}}$ before the ageing test, from Table 1, is 1475.9/1693.4. This implies that $L_{a}$ before the thermal test, is $\sim 50 \mathrm{~nm}$. After the thermal test $L_{a}$ reduces to $\sim 44 \mathrm{~nm}$. From Figure 6, it can also be noted that both the $\mathrm{NiO}$ phonon at $524 \mathrm{~cm}^{-1}$ and $1100 \mathrm{~cm}^{-1}$ are asymmetrically broadened. Asymmetrical broadening is ascribed to phonon confinement [12], a phenomenon that results from reduction of particle sizes. This phenomenon is observed in particles with diameter between $5 \mathrm{~nm}$ and $20 \mathrm{~nm}$ [13-19]. The spectra confirm that the $\mathrm{NiO}$ particles are indeed nanosized probably less than $20 \mathrm{~nm}$ in diameter. It can be noted that the asymmetrical broadening is enhanced after the thermal ageing test as can 
be seen in Figure 6. Therefore, as much as the carbon grains reduce in size after the test, it appears that this is true for the $\mathrm{NiO}$ grains also, and that the particle sizes reduce to much less than $20 \mathrm{~nm}$ after the thermal test.

From Table 1, $\beta$ can be calculated using (7) thus: $\beta=$ $1-\left(\left(2 \times 10^{5}+1.5 \times 10^{5}\right) /\left(3.37 \times 10^{5}+2.16 \times 10^{5}\right)\right)=0.37$. Also, in the present study the proportion of carbon remaining in the sample after the test, $1-\beta$, from ( 8$)$, is $1-0.37=$ 0.63 and the photo-thermal conversion efficiency of the aged sample, from (4), $0.88-0.16=0.72$. This implies that the proportionality constant $K$ can be estimated to be $K \sim$ $0.63 / 0.72=0.875 \sim$ unity. This finding reveals the fact that the values of proportions of carbon remaining in the selective solar absorber after ageing at any given time determined from Raman spectroscopy can be used to estimate the absorber's performance at that time in terms of the photothermal conversion efficiency namely $\eta=\alpha_{\text {sol }}-\varepsilon_{\text {ther }}=(1 / K)(1-\beta)$.

Thermal ageing tests on five more samples and their corresponding Raman signatures are ongoing. These results will help in the further validation of the currently derived $\eta-\beta$ relationship.

\section{Conclusions}

Spectrally selective MWCNT/NiO nanocomposite coatings of varying CNT concentration were prepared on $\mathrm{Al}$ substrate by a sol-gel process. Solar absorption and thermal emission values as a function of CNT concentration have been presented. A typical sample was taken for accelerated thermal ageing test. Preliminary accelerated thermal ageing test on the sample showed that the composite material passes the test with PC value of -0.01 which is below the lower limit of 0.05 . Raman spectroscopy was used to estimate the proportions of carbon atoms lost to those that remain in the sample after the thermal ageing test. The proportion of carbon atoms that still remain in the sample after the thermal test is correlated to the photo-thermal conversion efficiency. More experimental results are underway to validate this first correlation and to determine the performance criterion limits from Raman spectra.

\section{List of Abbreviations and Nomenclature}

$\alpha_{\text {sol }}: \quad$ Normal solar absorptance

$\varepsilon_{\text {ther }}$ : Thermal emittance

$I_{\text {sol }}$ : $\quad$ Direct normal solar irradiance

$\eta$ : $\quad$ Photothermal conversion efficiency

PC: $\quad$ Performance criterion

f-MWCNT: Functionalized multiwall carbon nanotube

$\beta$ : $\quad$ The proportion of carbon remaining in the material

FEG-SEM: Field emission gun-scanning electron microscopy.

\section{Acknowledgments}

The authors acknowledge the financial assistance from the CSIR under these project numbers LHIE100 and LHTAE00.
K. Roro acknowledges the financial assistance from the CSIR for young researcher establishment funding (Project no.: YREF_2011_32) and B. Mwakikunga acknowledges the characterization facilities from Project number HGER27S.

\section{References}

[1] G. W. Crabtree and N. S. Lewis, "Solar energy conversion," Physics Today, vol. 60, no. 3, pp. 37-42, 2007.

[2] J. A. Duffie and W. A. Beckman, Solar Engineering of Thermal Processes, John Wiley \& Sons, New York, NY, USA, 1980.

[3] D. Katzen, E. Levy, and Y. Mastai, "Thin films of silica-carbon nanocomposites for selective solar absorbers," Applied Surface Science, vol. 248, no. 1-4, pp. 514-517, 2005.

[4] G. Katumba, L. Olumekor, A. Forbes et al., "Optical, thermal and structural characteristics of carbon nanoparticles embedded in $\mathrm{ZnO}$ and $\mathrm{NiO}$ as selective solar absorbers," Solar Energy Materials and Solar Cells, vol. 92, no. 10, pp. 1285-1292, 2008.

[5] G. Katumba, G. Makiwa, T. R. Baisitse, L. Olumekor, A. Forbes, and E. Wäckelgård, "Solar selective absorber functionality of carbon nanoparticles embedded in $\mathrm{SiO}_{2}, \mathrm{ZnO}$ and $\mathrm{NiO}$ matrices," Physica Status Solidi C, vol. 5, no. 2, pp. 549-551, 2008.

[6] Y. Mastasi, S. Polarz, and M. Antoriett, "Silica-carbon nanocomposite-a new concept for the design of solar absorbers," Advanced Functional Materials, vol. 12, no. 3, pp. 197-202, 2002.

[7] B. Carlsson, U. Frei, M. Kohl, and K. Moller, "Accelerated life testing of solar energy materials — case study of some selective materials for DHW systems," IEA SHCP Task X, 1994.

[8] K. T. Roro, N. Tile, B. Mwakikunga, B. Yalisi, and A. Forbes, "Solar absorption and thermal emission properties of multiwall carbon nanotube/nickel oxide nanocomposite thin films synthesized by sol-gel process," Materials Science and Engineering B, vol. 177, no. 8, pp. 581-587, 2012.

[9] A. C. Ferrari and J. Robertson, "Interpretation of Raman spectra of disordered and amorphous carbon," Physical Review $B$, vol. 61 , no. 20 , pp. $14095-14107,2000$.

[10] B. W. Mwakikunga, M. Maaza, K. T. Hillie, C. J. Arendse, T. Malwela, and E. Sideras-Haddad, "From phonon confinement to phonon splitting in flat single nanostructures: a case of $\mathrm{VO}_{2} @ \mathrm{~V}_{2} \mathrm{O}_{5}$ core-shell nano-ribbons," Vibrational Spectroscopy, vol. 61, pp. 105-111, 2012.

[11] F. Tuinstra and J. L. Koenig, "Raman Spectrum of Graphite," Journal of Chemical Physics, vol. 53, no. 3, pp. 1126-1130, 1970.

[12] H. Richter, Z. P. Wang, and L. Ley, "The one phonon Raman spectrum in microcrystalline silicon," Solid State Communications, vol. 39, no. 5, pp. 625-629, 1981.

[13] B. W. Mwakikunga, E. Sideras-Haddad, and M. Maaza, "First synthesis of vanadium dioxide by ultrasonic nebula-spray pyrolysis," Optical Materials, vol. 29, no. 5, pp. 481-487, 2007.

[14] B. W. Mwakikunga, A. Forbes, E. Sideras-Haddad, R. M. Erasmus, G. Katumba, and B. Masina, "Synthesis of tungsten oxide nanostructures by laser pyrolysis," International Journal of Nanoparticles, vol. 1, no. 3, pp. 185-202, 2008.

[15] B. W. Mwakikunga, A. Forbes, E. Sideras-Haddad, and C. Arendse, "Raman spectroscopy of $\mathrm{WO}_{3}$ nano-wires and thermo-chromism study of $\mathrm{VO}_{2}$ belts produced by ultrasonic spray and laser pyrolysis techniques," Physica Status Solidi A, vol. 205, no. 1, pp. 150-154, 2008.

[16] G. Katumba, B. W. Mwakikunga, and T. R. Mothibinyane, "FTIR and Raman spectroscopy of carbon nanoparticles in 
$\mathrm{SiO}_{2}, \mathrm{ZnO}$ and $\mathrm{NiO}$ matrices," Nanoscale Research Letters, vol. 3, no. 11, pp. 421-426, 2008.

[17] M. Govender, L. Shikwambana, B. W. Mwakikunga, A. Forbes, E. Sideras-Haddad, and R. M. Erasmus, "Esopen accessformation of tungsten oxide nanostructures by laser pyrolysis: stars, fibres and spheres," Nanoscale Research Letters, vol. 6, no. 1, article 166, 2011.

[18] R. Machaka, B. W. Mwakikunga, E. Manikandan, T. E. Derry, and I. Sigalas, "Raman spectrum of hot-pressed boron suboxide," Advanced Materials Letters, vol. 2, no. 1, p. 68, 2011.

[19] G. Goudec and Ph. Colamban, "Raman spectroscopy of nanomaterials: how spectra relate to disorder, particle size and mechanical properties," Progress in Crystal Growth and Characterization of Materials, vol. 53, no. 1, p. 56, 2007. 


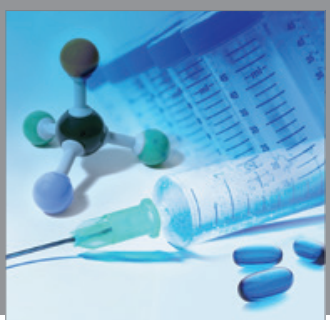

International Journal of

Medicinal Chemistry

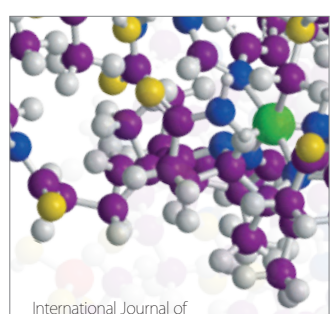

Carbohydrate Chemistry

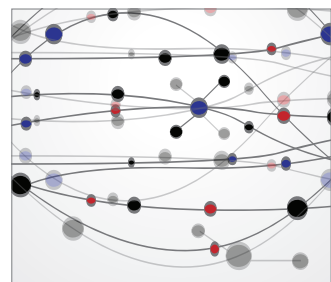

The Scientific World Journal
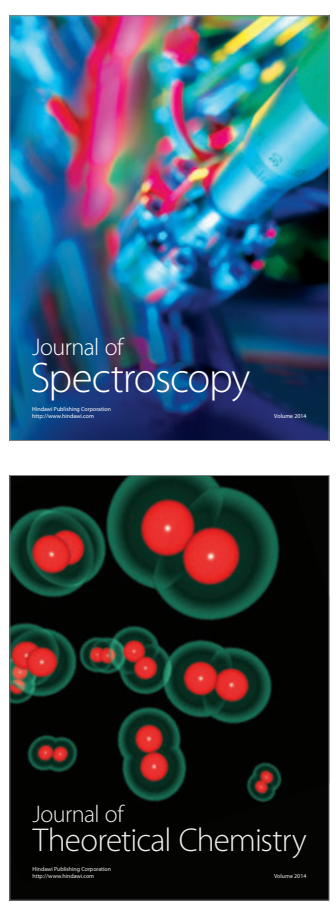
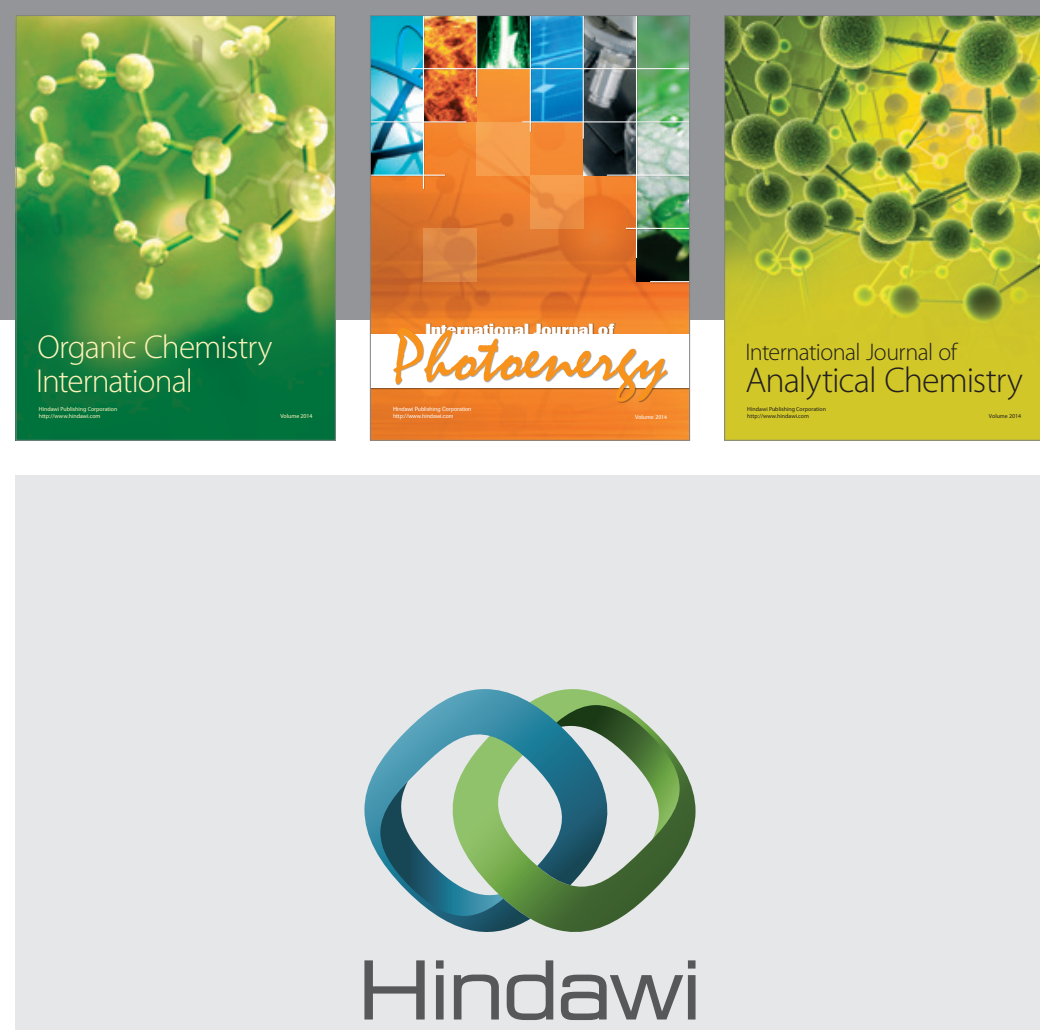

Submit your manuscripts at

http://www.hindawi.com
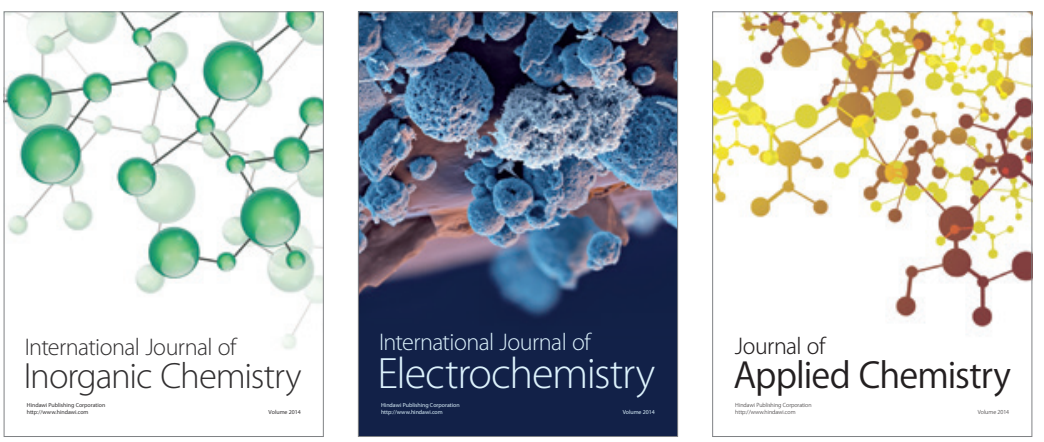

Journal of

Applied Chemistry
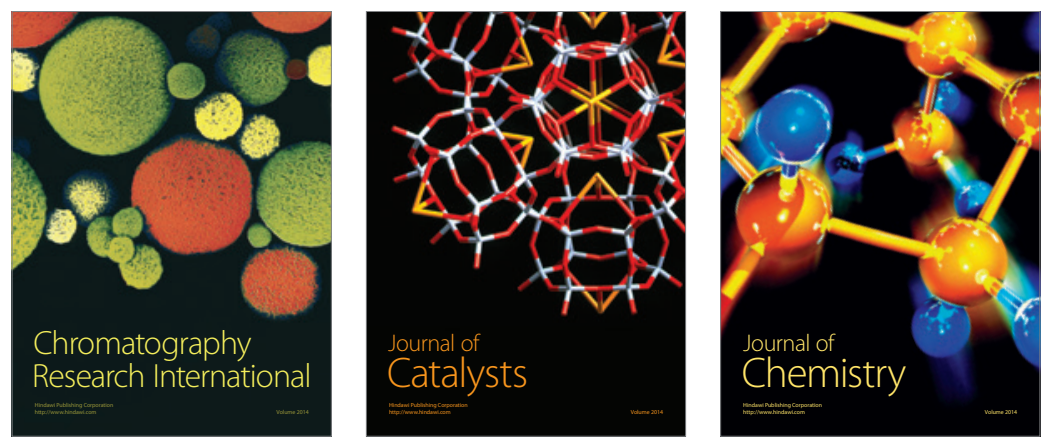
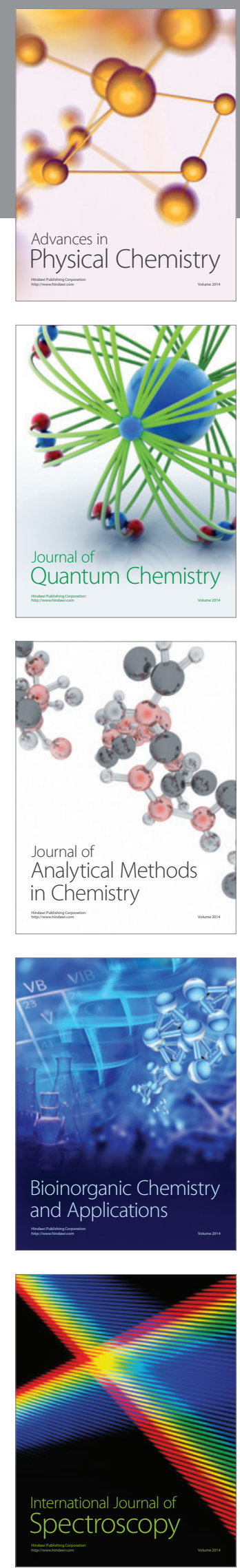